\title{
Correction to: Interplay Between Helicobacter pylori Infection, Interleukin-11, and Leukemia Inhibitory Factor in Gastric Cancer Among Egyptian Patients, by Sabry D, Abdelaleem OO, Hefzy EM, Ibrahim AA, Ahmed TI, Hassan EA, Abdel-Hameed ND, Khalil MAF. $\mathrm{J}$ Interferon Cytokine Res 2018;38(11):517-525. DOI: $10.1089 /$ jir.2018.0065
}

\footnotetext{
IN THe November 2018 Issue of Journal of Interferon \& Cytokine Research (vol. 38, no. 11, pp. 517-525), the article 1 "Interplay Between Helicobacter pylori Infection, Interleukin-11, and Leukemia Inhibitory Factor in Gastric Cancer Among Egyptian Patients," by Prof. Dina Sabry et al., Dr. Omayma O. Abdelaleem's affiliation, due to an error in production, was listed incorrectly. Dr. Abdelaleem's affiliation had been listed as:

Department of Medical Biochemistry and Molecular Biology, Faculty of Medicine, Cairo University, Cairo, Egypt.

The affiliation should read:

Department of Medical Biochemistry and Molecular Biology, Faculty of Medicine, Fayoum University, Fayoum, Egypt.

The online version of the article has been corrected to reflect this change.

The Publisher regrets this error and apologizes to the author.
} 